\title{
Burr-Assisted Anterior Lamellar Keratoplasty (DALK) in Patients with Corneal Stromal Scarring: A Case Series
}

\author{
George D. Kymionis - Michael A. Grentzelos • Myrsini Petrelli • \\ Clarice Giacuzzo · Konstantinos Droutsas
}

Received: February 3, 2020 / Published online: April 11, 2020

(C) The Author(s) 2020, corrected publication 2021

\section{ABSTRACT}

Purpose: To describe a modified technique of deep anterior lamellar keratoplasty (DALK) assisted by diamond ophthalmic burr (DOB) in two patients with corneal stromal scarring.

Methods: Two patients with corneal stromal scarring underwent a modified DALK technique with corneal stromal polishing assisted by a DOB until the level of the Descemet membrane. Results: There were no intra- or postoperative complications. Six months postoperatively, the corneal graft was clear, while corrected distance visual acuity improved in both cases.

Conclusions: Burr-assisted DALK seems to represent an alternative surgical technique in

Digital Features To view digital features for this article go to https://doi.org/10.6084/m9.figshare.12001326.

Electronic supplementary material The online version of this article (https://doi.org/10.1007/s40123020-00248-8) contains supplementary material, which is available to authorized users.

G. D. Kymionis $(\bowtie) \cdot$ M. A. Grentzelos · M. Petrelli

. C. Giacuzzo

Department of Ophthalmology, University of Lausanne, Jules-Gonin Eye Hospital, Fondation Asile des aveugles, Lausanne, Switzerland e-mail: gkymionis@gmail.com

\section{K. Droutsas}

First Department of Ophthalmology, National and Kapodistrian University of Athens, General Hospital "G. Gennimatas", Athens, Greece patients with corneal stromal scarring.Keywords: Corneal stromal scarring; Deep anterior lamellar keratoplasty (DALK); Diamond ophthalmic burr (DOB); Modified surgical technique

\section{Key Summary Points}

Deep anterior lamellar keratoplasty (DALK) is considered the procedure of choice in patients with corneal pathology not involving the endothelium.

Several modifications of DALK have been described for lamellar dissection and removal of the corneal stroma with the Anwar big-bubble (BB) technique being the most popular, while manual lamellar dissection has been reported to be the preferable surgical technique in cases of corneal stromal scars.

In this report, authors present two patients with corneal stromal scarring that underwent a modified DALK technique with corneal stromal polishing assisted by a diamond ophthalmic burr (DOB) until the level of Descemet membrane.

Burr-assisted DALK could represent an alternative surgical technique in patients with corneal stromal scarring. 


\section{INTRODUCTION}

Deep anterior lamellar keratoplasty (DALK) is considered the procedure of choice in patients with corneal pathology not involving the endothelium, including keratoconus, stromal dystrophies and scars [1]. Several modifications of DALK have been described for lamellar dissection and removal of the corneal stroma with the Anwar big-bubble (BB) technique being the most popular [1-9]. DALK with manual lamellar dissection has been reported to be the preferable surgical technique in cases of corneal stromal scars [1]. Herein, we describe a modified technique of DALK assisted by a diamond ophthalmic burr (DOB) in two patients with corneal stromal scarring.

\section{CASES PRESENTATION}

\section{Case 1}

An 86-year-old male was referred to our institute for DALK in his left eye. Past ocular history consisted of bilateral radial keratotomy (RK) in 1989 followed by bilateral cataract surgery in 1999. Moreover, he had undergone bilateral selective laser trabeculoplasty for open-angle glaucoma and intravitreal antivascular endothelial growth factor injections and panretinal photocoagulation for diabetic retinopathy (DR). His medical history was remarkable for multiple cardiovascular comorbidities, renal insufficiency and type 2 diabetes, and the patient was under anticoagulation therapy.
Corrected distance visual acuity (CDVA) was $20 / 200$ and intraocular pressure (IOP) was $13 \mathrm{mmHg}$ in his left eye. Slit-lamp examination revealed deep RK stromal scars and central anterior stromal scarring (Fig. 1a). The anterior segment was otherwise normal with a peripheral iridotomy and a posterior chamber intraocular lens. Fundus examination revealed a photocoagulated retina and myopia-relative degenerative changes. Endothelial cell density (ECD) was 1771 cells $/ \mathrm{mm}^{2}$ with a corneal pachymetry of $591 \mu \mathrm{m}$. The patient underwent a modified DALK assisted by a DOB.

The procedure was performed under retrobulbar anesthesia with $0.5 \%$ bupivacaine and $2 \%$ lidocaine. After the center of the host cornea was marked, the donor cornea was trephined at a diameter of $7.75 \mathrm{~mm}$ (Video 1). A Moria single-use adjustable-depth trephine (Moria Surgical, Antony, France) was used for partialthickness trephination of the host cornea at a depth of $550 \mu \mathrm{m}$ and a diameter of $7.50 \mathrm{~mm}$ to encompass as much scar tissue as possible with a residual corneal tissue of about $200 \mu \mathrm{m}$. A manual lamellar dissection was then performed using a crescent blade and a Tan lamellar dissector (ASICO, Westmont, IL, USA) initiating the procedure at 12 o'clock and meticulously excising the lamellae to control the depth of the dissection and stay within the lamellar plane. A handheld battery-driven DOB (Katena Eye Instruments, Denville, NJ) was then used to remove the remaining central and paracentral posterior stroma until the exposure of the Descemet membrane (DM) and polish the areas of deep corneal stromal scars (Fig. 1b). A careful, manual dissection was then performed to clear any residual stromal tissue at the periphery

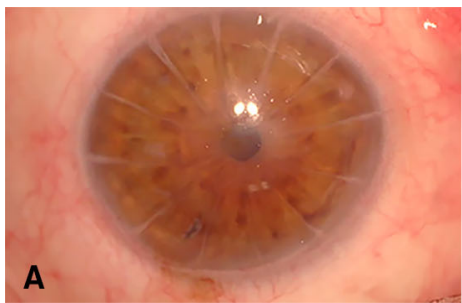

Fig. 1 Preoperative photo of the cornea (a); intraoperative photo showing the diamond ophthalmic burr removing the remaining posterior corneal stromal tissue until the
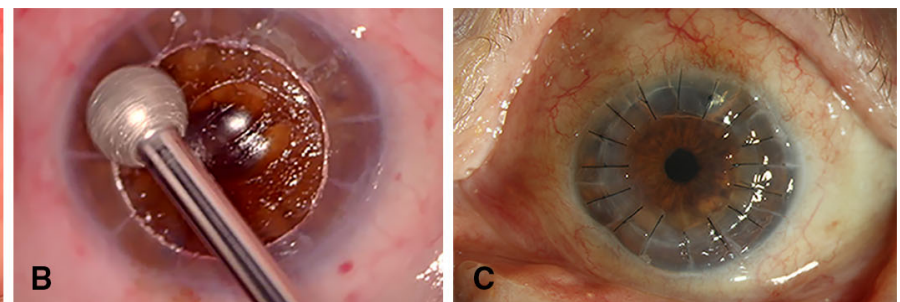

exposure of the Descemet membrane (b); postoperative photo of the cornea $(\mathbf{c})$ 


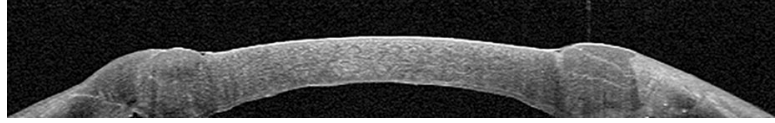

Fig. 2 Postoperative anterior segment optical coherence tomography showing the host Descemet membrane attached to the corneal stromal graft

where DOB smoothing was not possible. After removing the donor endothelium, the donor cornea was sutured on the host corneal bed using 16 interrupted 10-0 nylon sutures. Moxifloxacin (Vigamox, Alcon Laboratories, Inc.) eye drops were instilled, eye ointment tobramycin $0.3 \%$ with dexamethasone $0.1 \%$ (Tobradex, Alcon) was applied, and the eye was patched. Postoperative therapy included moxifloxacin (Vigamox, Alcon Laboratories, Inc) four times per day for 1 week and dexamethasone $0.1 \%$ (Dexafree, Thea Laboratories, Inc.) four times per day gradually tapered over 6 months.

Six months postoperatively, the corneal graft was clear with no signs of rejection or failure and a CDVA of 20/100 (Fig. 1c). ECD was 1651 cells $/ \mathrm{mm}^{2}$. There were no complications or adverse events during the follow-up period. Postoperative anterior segment optical coherence tomography (AS-OCT) (Avanti, OptovueInc, Fremont, CA, USA) showed the host DM attached to the corneal stromal graft (Fig. 2).

Written informed consent for publication of the patient's clinical details was obtained.

\section{Case 2}

A 51-year-old male was referred to our institute for the management of a corneal scar in his left eye. He reported a corneal injury from a metallic foreign body 4 months before presentation. His medical history included type 1 diabetes. CDVA was 20/160, and IOP was $17 \mathrm{mmHg}$. Slit-lamp examination and AS-OCT revealed anterior corneal stromal scarring involving the visual axis (Fig. 3); the anterior segment was otherwise normal. Fundus examination revealed signs of mild, non-proliferative DR. The patient was further followed for 6 months to evaluate any change in his corneal status before planning any intervention. A diagnostic rigid contact lens (RCL) fitting improved the patient's visual
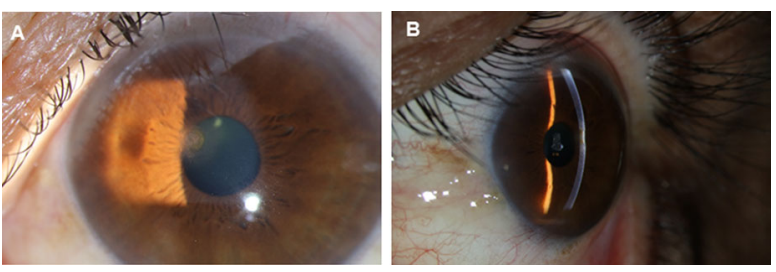

Fig. 3 Preoperative slit-lamp photos (a, b) depicting anterior corneal stromal scarring involving the visual axis

acuity significantly. For this reason, we initially proceeded with phototherapeutic keratectomy combined with corneal cross-linking as the ablation depth was high $(170 \mu \mathrm{m})$ and the fellow eye had a suspicious topography. However, during the postoperative period significant deep corneal stromal haze developed and CDVA decreased to counting fingers. ECD was 2539 cells $/ \mathrm{mm}^{2}$ with a corneal pachymetry of $375 \mu \mathrm{m}$. Due to the persistent aforementioned corneal stromal haze and patient's reluctance to take RCL daily, we decided to perform DALK. An uneventful modified DALK assisted by a DOB was performed as described previously.

Six months postoperatively, the corneal graft was clear with no signs of rejection or failure (Fig. 4). CDVA improved to 20/100 with presence of significant postoperative suture-related astigmatism, while ECD was 1582 cells $/ \mathrm{mm}^{2}$. There were no complications or adverse events during the follow-up period.

Written informed consent for publication of the patient's clinical details was obtained.

\section{DISCUSSION}

DALK represents a good alternative surgical technique to penetrating keratoplasty (PKP) in cases with normal endothelium [1]. It involves
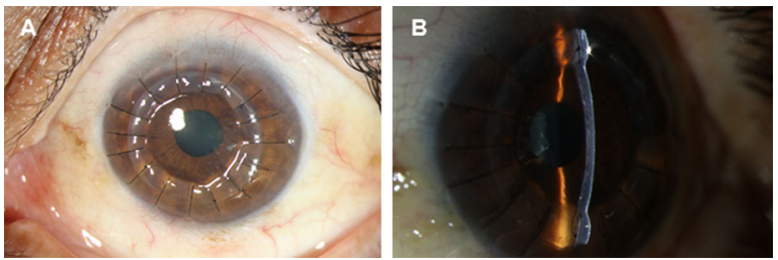

Fig. 4 Six-month postoperative slit-lamp photos (a, b) showing a clear and attached graft 
the removal of corneal stroma, while leaving host endothelium and DM intact [1]. Several techniques and modifications of DALK have been described to accomplish removal of the corneal stroma by means of lamellar dissection [1-9]. Among all the techniques described, the most popular is the BB [1-9]. The most common complication of the BB technique is the formation of large DM perforation that requires conversion to PKP [1-3]. The risk of intraoperative perforation is higher in case of corneal stromal scars [1]. Moreover, corneal stromal scarring may impede the lamellar dissection and DM separation during the $\mathrm{BB}$ technique resulting in unsuccessful BB formation. Reinhart et al. reported that the $\mathrm{BB}$ technique may carry higher risk for perforation in eyes with residual stromal scarring down to the DM, and other DALK techniques should be considered in these cases [1].

In 1999, Melles et al. described a manual dissection technique to visualize the corneal thickness by filling the anterior chamber with air, so that the depth of an instrument relative to the posterior corneal surface can be seen during surgery [4]. In a refinement of this technique, a dissection with viscoelastic was performed to separate the DM from the posterior stroma [5]. DALK with manual lamellar dissection has been reported to be a preferable surgical alternative for cases with corneal stromal scars, such as herpetic corneal scars, keratoconic patients with previous hydrops or those who have undergone RK [1, 6-9]. In 2009, Leccisotti reported the results of air-assisted manual DALK in eyes with herpetic corneal scars [6]. Vajpayee et al. described a knife-assisted DALK technique and showed it was effective for the management of keratoconus cases, three of which had healed hydrops [7]. Einan-Lifshitz et al. presented the outcomes of DALK in a group of ten patients who had undergone RK [8]. In their case series, BB creation was successful in only $10 \%$, while manual lamellar dissection was successfully performed in 60\%; there was a conversion to PKP due to a large DM perforation in the rest $30 \%$ of the eyes [8]. Einan-Lifshitz et al. concluded that DALK by manual lamellar dissection should be considered as a preferable surgical approach in post-RK patients to decrease the risk of perforation due to the corneal stromal RK scars [8]. Recently, in the study of Ucgul et al., a modified manual DALK technique using blunt scissors for lamellar dissection had similar clinical findings and results with BB [9]. However, with these techniques, the lamella is manually dissected until a pre- or near-DM layer is reached; the aim is to remove the stromal tissue until the level of DM but this increases the risk of perforation.

Herein, we describe a modified DALK technique with manual lamellar dissection assisted by DOB in two patients with corneal stromal scarring. The DOB has been used for the removal of superficial corneal opacities and regularization of the anterior corneal surface for the treatment of recurrent corneal erosions and anterior basement membrane dystrophy [10]. In our cases, the DOB was used to remove any remaining posterior corneal stroma, mainly centrally and paracentrally, and reach the DM level without perforation. It seems that the rotational movement of the DOB has a low risk of perforation, even when performed on an extremely thin residual stromal bed. We did not attempt the $\mathrm{BB}$ technique since its failure would possibly create DM tears or other corneal stromal tissue irregularities that might increase the difficulty of manual lamellar dissection and decrease the effectiveness of the modified DALK technique.

\section{CONCLUSIONS}

Burr-assisted DALK was shown to be safe and effective for manual lamellar dissection in our two cases. This modified DALK technique can be considered an alternative option in cases with corneal stromal scars. Large case series with long follow-up are necessary to thoroughly evaluate this surgical procedure and its outcomes mainly in challenging eyes with corneal stromal scarring.

\section{ACKNOWLEDGEMENTS}

Funding. No funding or sponsorship was received for this study or publication of this article. 
Authorship. All named authors meet the International Committee of Medical Journal Editors (ICMJE) criteria for authorship for this article, take responsibility for the integrity of the work as a whole, and have given their approval for this version to be published.

Disclosures. George D. Kymionis, Michael A. Grentzelos, Myrsini Petrelli, Clarice Giacuzzo, and Konstantinos Droutsas declare that they have no conflict of interest. Michael A. Grentzelos was granted a Scholarship by the Hellenic Society of Intraocular Implant and Refractive Surgery. The authors have no financial or proprietary interest in any materials or methods described herein. George D. Kymionis is a member of the journal's Editorial Board.

Compliance with Ethics Guidelines. Written informed consent for publication of both patient's clinical details was obtained.

Data Availability. Data sharing is not applicable to this article as no datasets were generated or analyzed during the current study.

Open Access. This article is licensed under a Creative Commons Attribution-NonCommercial 4.0 International License, which permits any non-commercial use, sharing, adaptation, distribution and reproduction in any medium or format, as long as you give appropriate credit to the original author(s) and the source, provide a link to the Creative Commons licence, and indicate if changes were made. The images or other third party material in this article are included in the article's Creative Commons licence, unless indicated otherwise in a credit line to the material. If material is not included in the article's Creative Commons licence and your intended use is not permitted by statutory regulation or exceeds the permitted use, you will need to obtain permission directly from the copyright holder. To view a copy of this licence, visit http://creativecommons.org/licenses/by$\mathrm{nc} / 4.0 /$.

\section{REFERENCES}

1. Reinhart WJ, Musch DC, Jacobs DS, Lee WB, Kaufman SC, Shtein RM. Deep anterior lamellar keratoplasty as an alternative to penetrating keratoplasty: a report by the American Academy of Ophthalmology. Ophthalmology. 2011;118:209-18.

2. Anwar M, Teichmann KD. Big-bubble technique to bare Descemet's membrane in anterior lamellar keratoplasty. J Cataract Refract Surg. 2002;28: 398-403.

3. Sarnicola V, Toro P, Gentile D, Hannush SB. Descemetic DALK and predescemetic DALK: outcomes in 236 cases of keratoconus. Cornea. 2010;29:53-9.

4. Melles GR, Lander F, Rietveld FJ, Remeijer L, Beekhuis WH, Binder PS. A new surgical technique for deep stromal, anterior lamellar keratoplasty. $\mathrm{Br} \mathrm{J}$ Ophthalmol. 1999;83:327-33.

5. Melles GR, Remeijer L, Geerards AJ, Beekhuis WH. A quick surgical technique for deep, anterior lamellar keratoplasty using visco-dissection. Cornea. 2000;19:427-32.

6. Leccisotti A. Air-assisted manual deep anterior lamellar keratoplasty for treatment of herpetic corneal scars. Cornea. 2009;28:728-31.

7. Vajpayee RB, Maharana PK, Sharma N, Agarwal T, Jhanji V. Diamond knife-assisted deep anterior lamellar keratoplasty to manage keratoconus. J Cataract Refract Surg. 2014;40:276-82.

8. Einan-Lifshitz A, Belkin A, Sorkin N, Mednick Z, Boutin T, Kreimei M, Chan CC, Rootman DS. Evaluation of big bubble technique for deep anterior lamellar keratoplastyin patients with radial keratotomy. Cornea. 2019;38:194-7.

9. Ucgul AY, Yesilirmak N, Yuvarlak A, Aydin B, Ozmen MC, Akata F. Blunt scissors stromal dissection technique compared with big-bubble deep anterior lamellar keratoplasty. Eye Contact Lens. 2019;45:195-200.

10. Vo RC, Chen JL, Sanchez PJ, Yu F, Aldave AJ. Longterm outcomes of epithelial debridement and diamond burr polishing for corneal epithelial irregularity and recurrent corneal erosion. Cornea. 2015;34:1259-65. 